\title{
Oportunidades para los agronegocios cooperativos costarricenses
}

\author{
Federico Li-Bonilla ${ }^{1}$ / Monserrat Espinach-Rueda ${ }^{2} /$ \\ Oscar Sanabria-Garro ${ }^{3}$
}

Escuela de Ciencias de la Administración Universidad Estatal a Distancia, Costa Rica

DOI:

Recepción: 10/07/2021 Aceptación: 20/08/2021

\section{Resumen}

Se ha realizado una investigación acción a través de un modelo de spin-off social planificado con el objetivo de crear start-ups, y con el fin de generar innovación social para la Universidad Estatal a Distancia. Este valor creado en la sociedad a través del sector cooperativo, produce trabajos directos e indirectos. Los acompañamientos en asesoría y auditoría a las cooperativas, por medio de los estudiantes, han proporcionado muchas mejoras en los problemas de gestión y buenas prácticas para optimizar los procesos productivos de las mismas.

Cada acompañamiento tiene un costo entre $\$ 100,000$ y $\$ 150,000$ y se cuenta con más de 26 profesionales ejecutando este trabajo, logrando impactar aproximadamente a 245 asociados y sus familias. Con esto se elimina ineficiencias, se logran precios justos y con el tiempo se estaría generando una curva de aprendizaje muy acelerada que ayude a los cooperativistas y a la región.

Palabras clave: Spin-off, innovación social, cooperativismo, agronegocios.

\begin{abstract}
An action research has been carried out through a planned social spin-off model with the aim of creating start-ups, and in order to generate social innovation for the University. This value created in society through the cooperative sector produces direct and indirect jobs. The advisory and auditing accompaniments to cooperatives, through students, have provided many improvements in management problems and good practices to optimize their productive processes.
\end{abstract}

Each accompaniment has a cost between $\$ 100,000$ and $\$ 150,000$ and there are more than 26 professionals executing this work, managing to impact approximately 245 associates and their families. With this, inefficiencies are eliminated, fair prices are achieved and, over time, a very accelerated learning curve will be generated that helps cooperative members and the region.

Key words: Spin-off, social innovation, cooperativism, agribusiness.

1. Doctor en Ciencias de la Administración, Universidad Estatal a Distancia, Director Escuela de Ciencias de la Administración email: fli@uned.ac.cr, ORCID: https://orcid.org/0000-0002-6390-1194

2. Doctora en Ciencias Económicas y Administrativas, Universidad Estatal a Distancia, Profesora Escuela de Ciencias de la Administración, email: mespinach@uned.ac.cr, ORCID: https://orcid.org/0000-0002-2681-4884

3. Doctor en Ciencias Empresariales, Universidad Estatal a Distancia, Profesor Escuela de Ciencias de la Administración , email: osanabria@uned.ac.cr, ORCID: https://orcid.org/0000-0002-7754-7130 


\section{Introducción}

Las empresas cooperativas consideradas en este estudio tienen un común denominador centrado en problemas de gestión, que van desde la falta de definición de su oferta de valor, hasta uso inadecuado de recursos que comprometen la sobrevivencia y el patrimonio de los asociados, lo que ha llevado a la paralización de la empresa con riesgo inminente de cierre. Consolidar el entendimiento de la oferta de valor en cada una de las empresas cooperativas permitirá redefinir su mapa estratégico que potencie el logro de los objetivos de creación y consolide la asociatividad de sus bases.

La Escuela de Ciencias de la Administración de la Universidad Estatal a Distancia $(\mathrm{UNED})^{4}$ pretende brindar las herramientas de gestión necesarias para reactivar las operaciones de las empresas cooperativas contempladas en este estudio, de forma tal que sus órganos de administración y asociados puedan redefinir sus objetivos empresariales, generando el cambio, cultura y documentación necesaria que permita lograr el apoyo de sus grupos de interés de naturaleza bancaria $\mathrm{y}$ de fomento cooperativo que permita reactivarlas. El estudio contempla tres empresas cooperativas del sector de agronegocios que pertenecen a sectores muy importantes de la base productiva del país y de sus territorios.
Una propuesta de valor, robusta y permeable a sus asociados, clientes y grupos de interés en adición a una gestión apropiada y una estrategia de capitalización sostenible, conduce a la salud financiera y solidez patrimonial de la cooperativa, fundamental para su sostenibilidad, siendo el objetivo principal del rescate empresarial de estas organizaciones.

El fundamento metodológico para el abordaje de la problemática empresarial descrito corresponde a un spin-off social planificado que persigue no solo el rescate en condiciones de inviabilidad empresarial, sino que también apoya el surgimiento de otros grupos empresariales cooperativos. Se parte de un entendimiento de la situación organizacional agregada, para luego segmentar en aspectos relacionados con bases asociativas, los proyectos productivos, la situación financiera, los fundamentos y realidad de los medios de vida de los asociados como catalizadores de identidad y cooperación para la reactivación de su empresa, todo conformando el modelo de negocios de la cooperativa; así como la configuración de los modelos de producción que potencian sus proyectos.

Toda vez que los signos vitales gerenciales, así como la base asociativa ha retomado sus compromisos financieros y asociativos, en forma paralela, la UNED provee de los recursos profesionales y logísticos necesarios para reforzar las áreas funcionales y procesos

4. Los autores harán referencia a la institución por medio de sus siglas, UNED. 
gerenciales de la cooperativa hasta lograr suficiencia en liquidez -punto de equilibrio- y rentabilidad. El spin-off social planificado tiene una duración de hasta 6 años y espera apoyar al menos 32 organizaciones.

\section{Entorno y problema}

El rescate empresarial se ha llevado a cabo en tres agronegocios cooperativos, cuya descripción se muestra en la tabla 1:

\section{Tabla 1}

Localización y giro de los agronegocios rescatados

\begin{tabular}{lclcc}
\hline \multicolumn{1}{c}{ Cooperativa } & Ubicación & \multicolumn{1}{c}{ Giro } & $\begin{array}{c}\text { Número de } \\
\text { asociados }\end{array}$ & $\begin{array}{c}\text { Fecha de } \\
\text { incorporación }\end{array}$ \\
\hline CoopeChorotega & $\begin{array}{l}\text { Santa Cruz, } \\
\text { Guanacaste }\end{array}$ & $\begin{array}{l}\text { Ganadería de } \\
\text { carne y } \\
\text { servicios } \\
\text { múltiples } \\
\text { Producción y } \\
\text { procesamient } \\
\text { Norte }\end{array}$ & 83 & 2015 \\
CoopeLacteos & $\begin{array}{l}\text { Upala, } \\
\text { Alajuela }\end{array}$ & $\begin{array}{l}\text { Pdeche } \\
\text { Producción y } \\
\text { procesamient } \\
\text { o de cacao }\end{array}$ & 107 & 2016 \\
& Upala, & & \\
\hline
\end{tabular}

Un factor común de Coopechorotega y CoopeCacao ha sido una operación limitada a la luz del proyecto descrito en el documento constitutivo PVU (Posibilidad, Viabilidad, Utilidad) requerido como requisito esencial por las autoridades de fomento cooperativo y normativas del Ministerio de Trabajo y Seguridad Social para formalizar la personaría jurídica de cada grupo a constituirse. No se generaron operaciones a la luz del proyecto lo que impidió cumplir su objetivo constitutivo, ambas por una configuración inapropiada de su proyecto productivo caracterizada por el sobredimensionamiento en función de su experiencia y capacidad patrimonial. Esto generó problemas legales y operativos en general, que las llevó prácticamente a la extinción.

Un caso diferente al anterior es el de CoopeLacteos, cooperativa que pudo ejecutar su proyecto constitutivo, pero los problemas de gestión han sido el génesis, así como las limitaciones impuestas por la pandemia COVID-19, que la han llevado prácticamente al cierre técnico con una alta probabilidad de quiebra. 
La tabla 2 muestra una descripción del proyecto productivo de cada cooperativa con su requerimiento de inversión y el capital suscrito. Se evidencia una incongruencia entre el tamaño del proyecto expresado en el capital requerido y el capital suscrito. Esta es una circunstancia vista en muchas empresas cooperativas, que podría asociarse también al desajuste entre la experiencia gerencial requerida para un emprendimiento de este tamaño y la real disponible en los cuadros directivos de las cooperativas. La incapacidad de poder conseguir financiamientos, a la luz de lo expuesto, se justifica toda vez que los organismos de fomento y financieros analizan la solicitud, que no prospera en el tiempo, llevando a las organizaciones a una situación como la descrita de cierre técnico y antesala de quiebra.

\section{Tabla 2}

Proyectos productivos, requerimientos de inversión y capital de la cooperativa

\begin{tabular}{llll}
\hline Cooperativa & Proyecto cooperativo & $\begin{array}{l}\text { Capital } \\
\text { requerido }\end{array}$ & $\begin{array}{l}\text { Capital } \\
\text { suscrito }\end{array}$ \\
\hline CoopeChorotega & $\begin{array}{l}\text { Planta procesadora y } \\
\text { comercializadora de } \\
\text { carne bovina, ovina y } \\
\text { porcina en Santa }\end{array}$ & $\begin{array}{l}\text { C3,119 millones } \\
\text { de colones }\end{array}$ & $\begin{array}{l}\text { C7.9 millones } \\
\text { de colones }\end{array}$ \\
& $\begin{array}{l}\text { Cruz, Guanacaste } \\
\text { Planta procesadora } \\
\text { de lácteos }\end{array}$ & $\begin{array}{l}\text { C86.9 millones } \\
\text { de colones }\end{array}$ & $\begin{array}{l}\text { C21.7 millones } \\
\text { de colones }\end{array}$ \\
CoopeLacteos & $\begin{array}{l}\text { Planta procesadora } \\
\text { de cacao }\end{array}$ & $\begin{array}{l}\text { C 2.7 Millones } \\
\text { de colones }\end{array}$ & de colones \\
\hline
\end{tabular}

\section{Materiales y Métodos}

El fundamento metodológico para el abordaje de la problemática empresarial descrito corresponde a un spin-off social planificado que persigue no solo el rescate en condiciones de inviabilidad empresarial, sino que también apoya el surgimiento de otros grupos empresariales cooperativos. Se parte de un entendimiento de la situación organizacional agregada, para luego segmentar en aspectos relacionados con bases asociativas, los proyectos productivos, la situación financiera, los fundamentos y realidad de los medios de vida de los asociados como catalizadores de identidad y cooperación para la reactivación de su empresa. Los componentes anteriores, conforman el modelo de negocios apropiado a la cooperativa, así como la configuración de los modelos de producción que potencian sus proyectos. 
Esta es una investigación acción, un trabajo de investigación mixta de campo, un modelo spin-off social planificado, que busca crear innovación social y por medio de esto en los territorios más pobres de Costa Rica, ofreciendo acompañamiento a cooperativas hasta por 6 años, cada acompañamiento cuesta entre $\$ 100,000$ y $\$ 150,000$ con los cuales se está impactando alrededor de 245 familias y los trabajos indirectos que se generan.

A través de este proyecto se apoya en la mejora e innovación de los modelos que no son operativos o tienen problemas graves de gestión, tienen limitaciones porque tienen poco tiempo, además el financiamiento es totalmente gratuito.

\section{Caso CoopeChorotega}

a. Caracterización de los sistemas de producción animal de los socios, como base para:

i. Rediseñar la oferta de valor de la cooperativa.

ii. Identificación de oportunidades de negocio alineado a la oferta de valor y capacidad técnico-financiera de la cooperativa.

b. Propuesta Proyecto Cooperativo para la Innovación y la Sostenibilidad Ganadera de la Región Chorotega:

i. Componente almacén de suministro.

ii. Componente producción y comercialización de forrajes.

\section{Caso Coopelacteos}

a. Rediseñar la oferta de valor de la cooperativa.

b. Restructurar la contabilidad y el sistema de información financiera.

c. Determinación de los flujos de leche desde las fincas de los asociados.

d. Restructuración de los flujos de manufactura y recuperación de la capacidad productiva.

e. Identificación de oportunidades de negocio alineado a la oferta de valor y capacidad técnico-financiera de la cooperativa.

\section{Caso CoopeCacao}

a. Rediseñar la oferta de valor de la cooperativa.

b. Determinación de los flujos de cacao desde las fincas de los asociados.

c. Estudio de factibilidad para establecimiento de una planta de fermentación y secado de cacao adecuada a los flujos presentes y esperados de cacao.

d. Identificación de oportunidades de negocio alineado a la oferta de valor y capacidad técnico-financiera de la cooperativa. 


\section{Resultados y Discusión}

\section{Caso CoopeChorotega}

En el proceso de análisis y reflexión para derivar la oferta de valor de la cooperativa se formuló el proyecto cooperativo para la innovación y la sostenibilidad ganadera de la Región Chorotega que resume la estrategia de rescate de la cooperativa, con sus dos componentes principales: a. almacén de suministros y b. producción y comercialización de forrajes. (Ver figura 1)

\section{Figura 1}

Mapa conceptual del rescate de CoopeChorotega

\section{Caso CoopeLacteos}

El mapa conceptual que resume la estrategia para el rescate de la cooperativa se muestra en la figura 2. Se detalla cada uno de los componentes principales que integran y reconstruyen la base asociativa, el potencial productivo de los asociados, la eficiencia de la planta, así como el abordaje de los aspectos de liquidez y rentabilidad sostenible, en un marco de férreo control interno y ampliación de la oferta de valor de la cooperativa para sus asociados y las comunidades a las cuales sirve. Caso

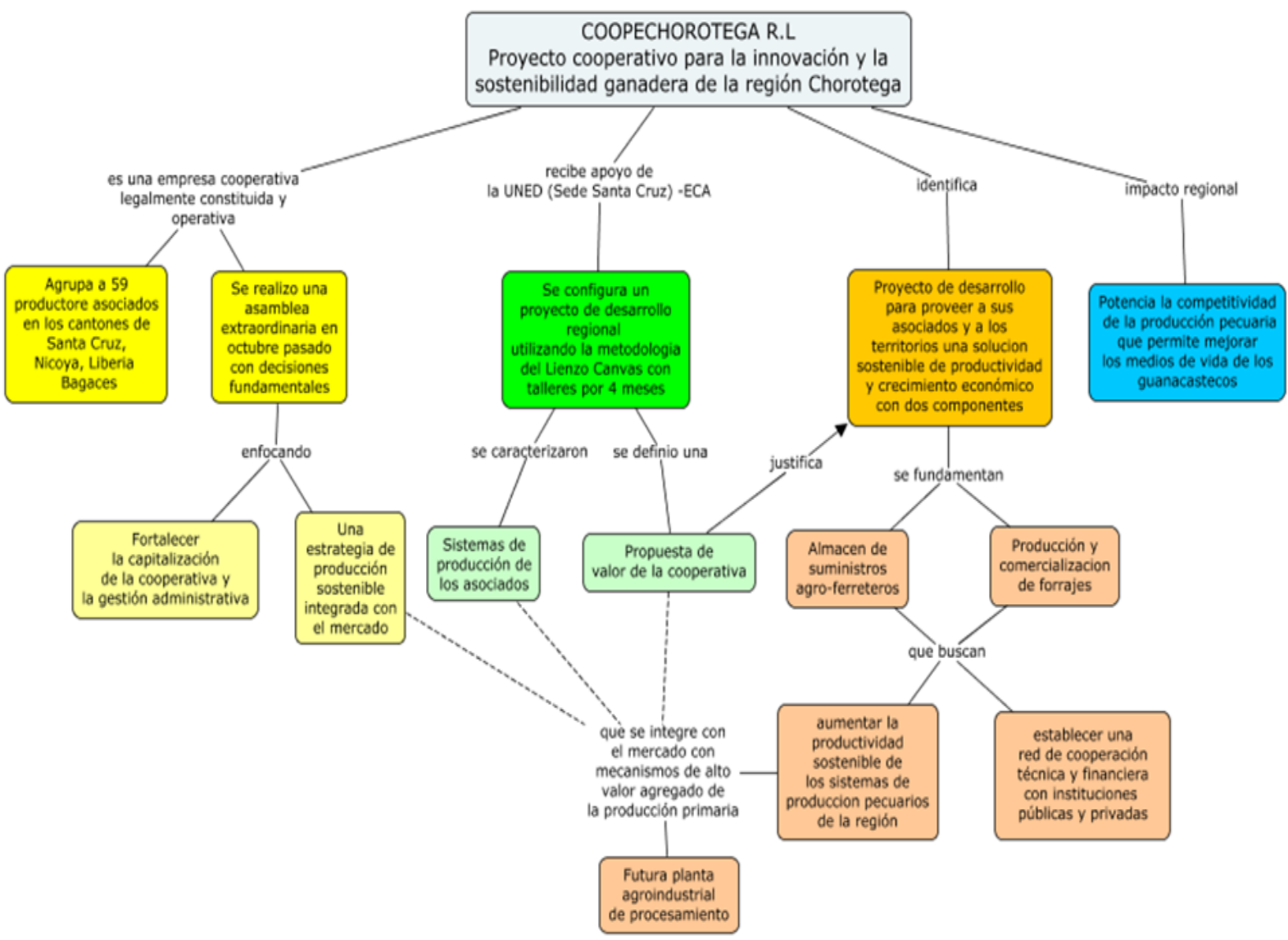




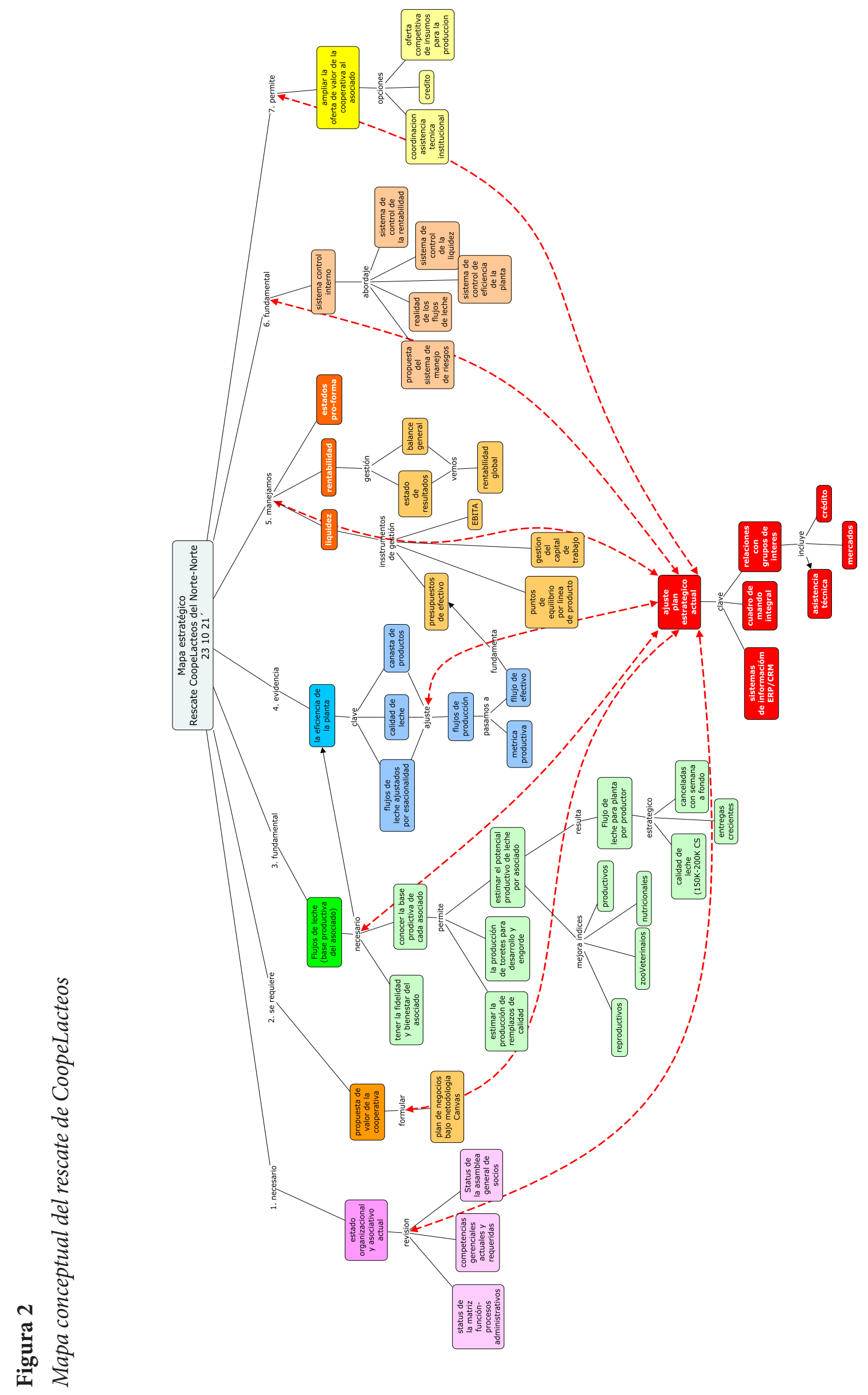




\section{CoopeCacao}

El abordaje del rescate de la cooperativa se fundamenta en 4 componentes principales:

a. el ordenamiento de la base asociativa,

b. la métrica productiva y el control de los costos,

c. la configuración de la planta procesadora (fermentado, secado, empaque) y d. el fortalecimiento de las redes y grupos de interés (ver figura 3).

Los componentes se concentraron en la mejora de las estructuras de gobernanza y salud financiera, formulación de la declaración de valor de cooperativa y el desarrollo de mercados de comercio justo de alto valor y reconocimiento de la calidad del cacao que se produce en el territorio.

\section{Figura 3}

Mapa conceptual del rescate de CoopeCacao

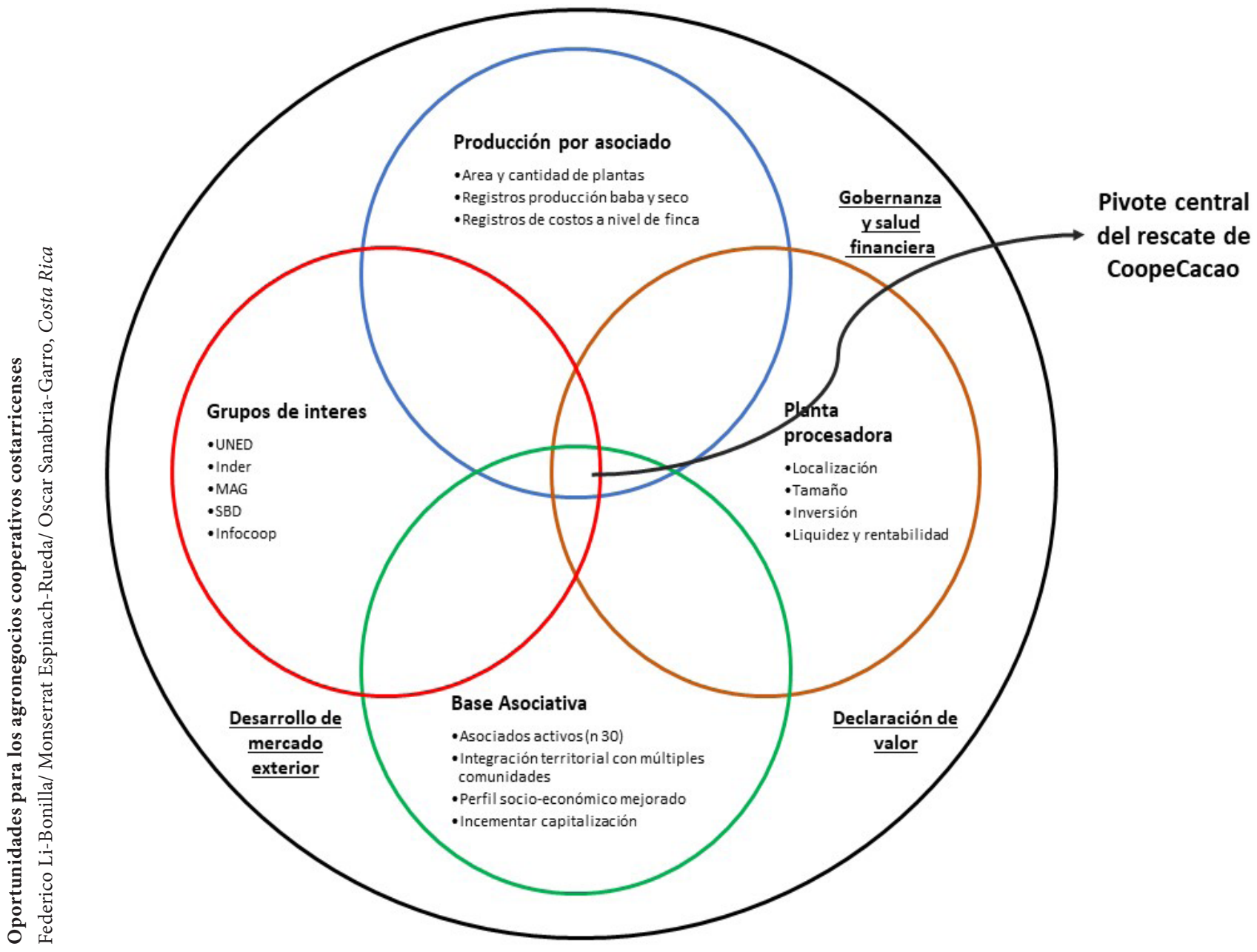




\section{Conclusiones}

En general, la necesidad que tienen las cooperativas de mejorar su gestión y buenas prácticas y un gran aprendizaje es evidente, por ello, la UNED por medio de un trabajo de consultoría y auditoría y mejora de gestión, de semana a semana ha hecho un aporte para que las cooperativas, que han estado a punto de cierre técnico, se puedan recuperar, planteando un nuevo modelo, plasmados en las figuras de este documento, que al aplicarse puede sacarlas hacia adelante en la zona de mayor depresión económica del país.

El Modelo ha sido ejecutado a través de un censo, que permite al auditarlas a todas, des- cubrir cómo recuperar a las Cooperativas y lo importante para la prosperidad de la sociedad es que cada vez van incrementando cooperativas en recuperación que ayudarán a muchas familias de las zonas más empobrecidas de Costa Rica.

Finalmente, este modelo de innovación social puede ser muy importante para las regiones latinoamericanas que han sido impactadas por el COVID-19 y los problemas históricos de gestión, mal manejo financiero y de falta de productividad al que se ve enfrentado el sector cooperativo en Latinoamérica.

\section{Referencias}

Espinach Rueda, M. (2018). Desarrollo sostenible para resguardar la seguridad humana, a partir de los resultados del índice de progreso social y su vinculación con la Economía social: Caso Costa Rica. Revista Espiga 17, (36), 159-175.

Espinach Rueda, M. (2018). Desarrollo Sostenible. Hacia el cumplimiento de la Agenda 2030 de la Organización de las Naciones Unidas. Caso Costa Rica. UNED, Costa Rica.

Gobierno de la República de Costa Rica y Programa de las Naciones Unidas para el Desarrollo. (2017). Costa Rica: Construyendo una visión compartida del desarrollo sostenible. Reporte Nacional Voluntario de los Objetivos de Desarrollo Sostenible. https://sustainabledevelopment.un.org/content/documents/15846Costa_Rica.pdf

Li Bonilla, F. y Espinach Rueda, M. (2020). "Economía Social, cooperativismo y la agenda 2030 de los Objetivos de Desarrollo Sostenible en la política de gestión de Costa Rica para crear desarrollo territorial”. Experiencias Emergentes de la Economía Social en Iberoamérica, OIBESCOOP. 76-96. 
Li Bonilla, F. y Espinach Rueda, M. (2021). "Modelo spin off social de empresas asociativas de base universitaria: caso UNED-Costa Rica, implementado en pandemia de la COVID-19". En Economía Social Solidaria y la COVID-19. Propuesta para una salida global de Gustavo Hernández Castro, Pablo Baisotti y Federico Li Bonilla, 277-303. UNED. 